UCD-00-11

gr-qc/0005017

May 2000

\title{
Logarithmic Corrections to Black Hole Entropy from the Cardy Formula
}

\author{
S. CARLIP ${ }^{\text {⿵ }}$ \\ Department of Physics \\ University of California \\ Davis, CA 95616 \\ USA
}

\begin{abstract}
Many recent attempts to calculate black hole entropy from first principles rely on conformal field theory techniques. By examining the logarithmic corrections to the Cardy formula, I compute the first-order quantum correction to the Bekenstein-Hawking entropy in several models, including those based on asymptotic symmetries, horizon symmetries, and certain string theories. Despite very different physical assumptions, these models all give a correction proportional to the logarithm of the horizon size, and agree qualitatively with recent results from "quantum geometry" in $3+1$ dimensions. There are some indications that even the coefficient of the correction may be universal, up to differences that depend on the treatment of angular momentum and conserved charges.
\end{abstract}

*email: carlip@dirac.ucdavis.edu 


\section{Introduction}

Recent progress in quantum gravity has made it possible for the first time to directly compute the Bekenstein-Hawking entropy of a black hole by counting microscopic states. Such calculations have been performed both in string theory [1] and in "quantum geometry" [2], and while significant questions remain in each approach, the outlook seems promising. The agreement between these very different approaches, and the agreement of both with semiclassical calculations that know nothing of the details of quantum gravity, has suggested that the asymptotic behavior of the density of states may be determined by some simple universal feature, such as the algebra of diffeomorphisms at the horizon [3, 田, 5, 6].

As Kaul and Majumdar have recently stressed [7], however, different quantum theories of gravity may lead to different higher order corrections to the Bekenstein-Hawking entropy. These corrections may display differences, or-less probably but more interestinglyrelations among quantizations. In Ref. [7], Kaul and Majumdar compute the lowest order corrections to the Bekenstein-Hawking entropy in a particular formulation [8] of the "quantum geometry" program of Ashtekar et al. They find that the leading correction is logarithmic, with

$$
S \sim \frac{A}{4 G}-\frac{3}{2} \ln \left(\frac{A}{4 G}\right)+\text { const. }+\ldots
$$

Although the existing computations of black hole entropy have very different physical starting points, most use techniques from two-dimensional conformal field theory at an intermediate stage. This may be no more than a useful trick - the Cardy formula [9, 10] makes it particularly easy to count states in a two-dimensional conformal field theory-but there are suggestions [11, 12] that such conformal field theories really provide a universal description of low-energy black hole thermodynamics. Whatever the origin of the conformal symmetry, however, the same trick that allows us to determine the asymptotic density of states also permits a direct computation of the leading quantum corrections to the Bekenstein-Hawking entropy.

In this paper, I compute the logarithmic terms in the Cardy formula and use the results to obtain quantum corrections to black hole entropy. I examine a number of approaches, including Strominger's asymptotic symmetry analysis [13 of the $(2+1)$-dimensional BTZ black hole [14]; the methods of Ref. [3, 4 , [], which are based on the behavior of symmetries at the black hole horizon in any dimension; and the string theoretical counting of D-brane states for BPS black holes [15, 16]. In all cases, I find qualitative agreement with the "quantum geometry" result (1.1), with answers that differ by a factor of two in the coefficient of the logarithm and (sometimes) an additional term that depends on conserved charges. I suggest that these differences may be traced back to an ambiguity in the treatment of angular momentum and other conserved quantities, which may lead to the counting of different sets of states. If this is the case, these results represent a surprising new universality in the logarithmic corrections to the Bekenstein-Hawking entropy. 


\section{Logarithmic Corrections to the Cardy Formula}

The recent "first principles" computations of black hole entropy, whatever their physical starting point, typically rely at some critical stage on the Cardy formula [9, 10] for the density of states in a two-dimensional conformal field theory. I will start by reviewing the derivation of this formula, in order to obtain logarithmic corrections to the density of states.

Let us begin with an arbitrary two-dimensional conformal field theory with central charge $c$, with the standard Virasoro algebra

$$
\begin{aligned}
& {\left[L_{m}, L_{n}\right]=(m-n) L_{m+n}+\frac{c}{12} m\left(m^{2}-1\right) \delta_{m+n, 0}} \\
& {\left[\bar{L}_{m}, \bar{L}_{n}\right]=(m-n) \bar{L}_{m+n}+\frac{c}{12} m\left(m^{2}-1\right) \delta_{m+n, 0}} \\
& {\left[L_{m}, \bar{L}_{n}\right]=0}
\end{aligned}
$$

for the generators $L_{n}, \bar{L}_{n}$ of holomorphic and antiholomorphic diffeomorphisms. The partition function on the two-torus of modulus $\tau=\tau_{1}+i \tau_{2}$ is defined to be

$$
Z(\tau, \bar{\tau})=\operatorname{Tr} e^{2 \pi i \tau L_{0}} e^{-2 \pi i \bar{\tau} \bar{L}_{0}}=\sum \rho(\Delta, \bar{\Delta}) e^{2 \pi i \Delta \tau} e^{-2 \pi i \bar{\Delta} \bar{\tau}} .
$$

For a unitary theory, $\rho$ is the number of states with eigenvalues $L_{0}=\Delta, \bar{L}_{0}=\bar{\Delta}$, as can be seen by inserting a complete set of states into the trace. For a nonunitary theory, $\rho$ is the difference between the number of positive- and negative-norm states with appropriate eigenvalues.

If we could somehow determine the partition function, we could extract the density of states by contour integration. Treat $\tau$ and $\bar{\tau}$ as independent complex variables (this is not necessary, but it simplifies the computation), and let $q=e^{2 \pi i \tau}$ and $\bar{q}=e^{2 \pi i \bar{\tau}}$. Then

$$
\rho(\Delta, \bar{\Delta})=\frac{1}{(2 \pi i)^{2}} \int \frac{d q}{q^{\Delta+1}} \frac{d \bar{q}}{\bar{q}^{\bar{\Delta}+1}} Z(q, \bar{q}),
$$

where the integrals are along contours that enclose $q=0$ and $\bar{q}=0$. Of course, it is rare that we actually know $Z(q, \bar{q})$. But Cardy has shown that it is still possible to relate the behavior of the partition function at high "energy" to its simpler behavior at low "energy," thus giving us some control over the integral (2.3).

Cardy's basic result [9, 10] is that the quantity

$$
\operatorname{Tr} e^{2 \pi i\left(L_{0}-\frac{c}{24}\right) \tau} e^{-2 \pi i\left(\bar{L}_{0}-\frac{c}{24}\right) \bar{\tau}}=e^{\frac{\pi c}{6} \tau_{2}} Z(\tau, \bar{\tau})
$$

is modular invariant, and in particular invariant under the large diffeomorphism $\tau \rightarrow-1 / \tau$ that interchanges the circumferences of the torus. The argument is universal, involving only some general properties of conformal field theory. We can use this result to attempt to evaluate the integral (2.3) by steepest descent. To do so, let $\Delta_{0}$ be the lowest eigenvalue of $L_{0}$ (often but not always zero), and define

$$
\tilde{Z}(\tau)=\sum \rho(\Delta) e^{2 \pi i\left(\Delta-\Delta_{0}\right) \tau}=\rho\left(\Delta_{0}\right)+\rho\left(\Delta_{1}\right) e^{2 \pi i\left(\Delta_{1}-\Delta_{0}\right) \tau}+\ldots
$$


(For simplicity, I have suppressed the $\bar{\tau}$ dependence.) It is then straightforward to show that

$$
\rho(\Delta)=\int d \tau e^{-2 \pi i \Delta \tau} e^{-2 \pi i \Delta_{0} \frac{1}{\tau}} e^{\frac{2 \pi i c}{24} \tau} e^{\frac{2 \pi i c}{24} \frac{1}{\tau}} \tilde{Z}(-1 / \tau)
$$

By construction, $\tilde{Z}(-1 / \tau)$ approaches a constant, $\rho\left(\Delta_{0}\right)$, for large $\tau_{2}$, so the integral (2.6) can safely be evaluated by steepest descents provided that the imaginary part of $\tau$ is large at the saddle point.

The integral we need has the form

$$
I[a, b]=\int d \tau e^{2 \pi i a \tau+\frac{2 \pi i b}{\tau}} f(\tau) .
$$

The argument of the exponent is extremal at $\tau_{0}=\sqrt{b / a}$, and expanding around this extremum, we find

$$
I[a, b] \approx \int d \tau e^{4 \pi i \sqrt{a b}+\frac{2 \pi i b}{\tau_{0}^{3}}\left(\tau-\tau_{0}\right)^{2}} f\left(\tau_{0}\right)=\left(-\frac{b}{4 a^{3}}\right)^{1 / 4} e^{4 \pi i \sqrt{a b}} f\left(\tau_{0}\right) .
$$

In particular, if $\Delta_{0}$ is small $\left(\Delta_{0} \ll c\right)$ and $\Delta$ is large, the integral (2.6) yields

$$
\rho(\Delta) \approx\left(\frac{c}{96 \Delta^{3}}\right)^{1 / 4} \exp \left\{2 \pi \sqrt{\frac{c \Delta}{6}}\right\}
$$

The exponential term in (2.9) gives the standard Cardy formula, but we have now found the leading correction as well.

We must next ask how reliable this approximation is. For $f(\tau)$ constant, the integral (2.7) can be performed explicitly, yielding a Bessel function, whose asymptotic behavior agrees with (2.8) with additional terms that are exponentially suppressed. Corrections from the nonconstancy of $f(\tau)$ may also be computed, and for $f(\tau)=\tilde{Z}(-1 / \tau)$, it is easy to check that these are again exponentially suppressed. For large $\Delta$, the expression (2.9) thus gives a reliable first-order correction to the standard Cardy formula.

It should be stressed that the central charge $c$ appearing in (2.9) is the full central charge of the conformal field theory. In general, $c$ will consist of a "classical" term $c_{\text {class }}$, which already appears in the Poisson brackets of the $L_{n}$, plus a quantum correction that can change the exponent in (2.9) from its "classical" value. In contrast to the prefactor in (2.9), this correction is likely to be highly model-dependent. Nevertheless, a bit can be said about its general features; see Appendix B.

\section{The BTZ Black Hole}

As our first application of Eqn. (2.9), let us evaluate the logarithmic corrections to Strominger's derivation of the entropy of the BTZ black hole. This $(2+1)$-dimensional

*See [17] for details. A closely related but more general result, obtained from a sophisticated number theoretical analysis that yields a much more complete description of the asymptotics, is discussed in [18]. 
black hole has a metric

$$
d s^{2}=-N^{2} d t^{2}+N^{-2} d r^{2}+r^{2}\left(d \phi+N^{\phi} d t\right)^{2}
$$

with

$$
N=\left(-8 G M+\frac{r^{2}}{\ell^{2}}+\frac{16 G^{2} J^{2}}{r^{2}}\right)^{1 / 2}, \quad N^{\phi}=-\frac{4 G J}{r^{2}} \quad(|J| \leq M \ell),
$$

and solves the vacuum Einstein field equations with a cosmological constant $\Lambda=-1 / \ell^{2}$. The spacetime is thus asymptotically anti-de Sitter, and has an outer (event) and an inner horizon at

$$
r_{ \pm}^{2}=4 G M \ell^{2}\left\{1 \pm\left[1-\left(\frac{J}{M \ell}\right)^{2}\right]^{1 / 2}\right\}
$$

i.e.,

$$
M=\frac{r_{+}{ }^{2}+r_{-}^{2}}{8 G \ell^{2}}, \quad J=\frac{r_{+} r_{-}}{4 G \ell} .
$$

As Brown and Henneaux first noted [19], the asymptotic symmetries of Einstein gravity in $2+1$ dimensions with negative $\Lambda$ are described by a pair of Virasoro algebras, with central charges

$$
c=\bar{c}=\frac{3 \ell}{2 G} .
$$

Intuitively, these are the symmetries of the adS "cylinder at infinity," obtained by restricting diffeomorphisms in the bulk to those that preserve adS boundary conditions. The central charges (3.5) are classical, but they will presumably be reflected in any quantum theory of gravity. Thus in any quantum theory of gravity that has the correct classical limit, the fields should transform under representations of these Virasoro algebras. Given some plausible assumptions [17] - for example, that $\Delta_{0}$ is small — one should therefore be able to use the Cardy formula to compute the asymptotic density of states.

Now, the generators of the Brown-Henneaux Virasoro algebras can be computed explicitly: they are simply the Hamiltonian and momentum constraints of general relativity smeared against appropriate vector fields. For the BTZ black hole, one finds that up to an ambiguous additive constant [20],

$$
\Delta=\frac{\left(r_{+}+r_{-}\right)^{2}}{16 G \ell}, \quad \bar{\Delta}=\frac{\left(r_{+}-r_{-}\right)^{2}}{16 G \ell} .
$$

As Strominger observed, Eqns. (3.5) and (3.6) can be used to evaluate the exponent in (2.9), yielding

$$
2 \pi \sqrt{\frac{c \Delta}{6}}+2 \pi \sqrt{\frac{\bar{c} \bar{\Delta}}{6}}=\frac{2 \pi r_{+}}{4 G},
$$

giving the standard Bekenstein-Hawking entropy for the (2+1)-dimensional black hole.

It is now easy to read off the logarithmic corrections to the entropy. From (2.9),

$$
\rho(\Delta, \bar{\Delta}) \approx \frac{8 G \ell^{2}}{\left(r_{+}^{2}-r_{-}^{2}\right)^{3 / 2}} \exp \left\{\frac{2 \pi r_{+}}{4 G}\right\} .
$$


Thus

$$
S \sim \frac{2 \pi r_{+}}{4 G}-\frac{3}{2} \ln \left(\frac{r_{+}^{2}-r_{-}^{2}}{G^{2}}\right)+\text { const. }=\frac{2 \pi r_{+}}{4 G}-\frac{3}{2} \ln \frac{2 \pi r_{+}}{G}-\frac{3}{2} \ln \kappa \ell+\text { const. }
$$

where

$$
\kappa=\frac{r_{+}^{2}-r_{-}^{2}}{\ell^{2} r_{+}}
$$

is the surface gravity. The logarithmic terms in (3.9) have the same form as those found by Kaul and Majumdar for the nonrotating $(3+1)$-dimensional black hole. Because of the term involving $\kappa$, however, the coefficients are different; in particular, if one restricts to zero angular momentum $\left(r_{-}=0\right)$, one finds logarithmic term that differs from (1.1) by a factor of two.

There is an alternative derivation of the BTZ black hole entropy, first proposed in Ref. 21], that directly counts states of an induced $\operatorname{SL}(2, \mathbb{R}) \times \operatorname{SL}(2, \mathbb{R})$ Wess-Zumino-Witten model at the black hole horizon. While the horizon radius $r_{+}$has a natural expression in such a WZW model, it is difficult to fix $r_{-}$, so one instead fixes a conjugate variable (essentially a component of the triad at the horizon). As explained in Appendix B of Ref. [17], the resulting partition function can be viewed as a functional Fourier transform of the partition function (2.2), where the central charge in $(2.2)$ for an $\operatorname{SL}(2, \mathbb{R})$ WZW model is $c \approx 3$. One finds

$$
Z=\sum_{N} \rho(N) \exp \left\{2 \pi i \tau\left(N-\frac{k^{2} r_{+}^{2}}{\ell^{2}}\right)\right\}
$$

with

$$
\rho(N) \sim \sum_{n=0}^{N} \rho_{0}(N) \rho_{0}(N-n),
$$

where $\rho_{0}$ is the partition function for an $\operatorname{SL}(2, \mathbb{R})$ WZW model and $k=\ell / 4 G$.

In the large $k$ limit, the three oscillators of $\operatorname{SL}(2, \mathbb{R})$ can be treated independently, and (2.9) gives

$$
\rho(N) \sim \sum_{n=0}^{N} N^{-3 / 4}(N-n)^{-3 / 4} \exp \{\sqrt{2} \pi(\sqrt{n}+\sqrt{N-n})\} .
$$

We can evaluate this expression by approximating the sum as an integral and using the method of steepest descents, obtaining

$$
\rho(N) \sim N^{-3 / 4} e^{2 \pi \sqrt{N}} .
$$

In the formalism of Ref. [21], the partition function (3.11) is subject to a physical state condition $\Delta=0$, that is, $N=k^{2} r_{+}^{2} / \ell^{2}$. We thus obtain a density of states

$$
\rho(N) \sim\left(r_{+} / G\right)^{-3 / 2} \exp \left\{\frac{2 \pi r_{+}}{4 G}\right\}
$$


The resulting logarithmic correction to the entropy agrees exactly with that of Kaul and Majumdar.

It is interesting to note that logarithmic corrections of this sort are absent in the Euclidean path integral approach to BTZ black hole entropy. The first-order corrections were calculated in that formalism in Ref. [22]; they give an exponentially suppressed contribution to the density of states, with no power law prefactor that would translate into a logarithmic correction to the entropy.

\section{Horizon Conformal Field Theory}

The conformal field theory derivations of the preceding section rely on special features of the $(2+1)$-dimensional black hole. The results are more general than they might appear at first sight, since many black holes in string theory have a near-horizon structure that looks like that of a BTZ black hole [1, 17]. Others do not, however, and the methods do not directly generalize directly to higher dimensions.

A different conformal field theory approach to black hole entropy has recently been proposed, based on a possible universal Virasoro algebra at the horizon [3, 4, 5]. This algebra is obtained by treating the horizon as a boundary and considering the behavior of the algebra of diffeomorphisms of the " $r-t$ plane" near the horizon. The proper choice of boundary conditions is not entirely clear, but several different choices give rise to a Virasoro algebra with central charge

$$
c=\frac{3 A}{2 \pi G} \frac{\beta}{\kappa}
$$

and an $L_{0}$ eigenvalue

$$
\Delta=\frac{A}{16 \pi G} \frac{\kappa}{\beta},
$$

where $A$ is the horizon area (in any dimension), $\kappa$ is the surface gravity, and $\beta$ is an undetermined periodicity. An analysis of the Liouville theory near the horizon obtained from dimensional reduction of Einstein gravity gives a similar result [6].

It is easy to see that these values of $c$ and $\Delta$, inserted into the Cardy formula, give the standard Bekenstein-Hawking entropy. But we can now go further, and compute the logarithmic corrections: Eqn. (2.9) yields

$$
\rho(\Delta) \sim \frac{c}{12}\left(\frac{A}{8 \pi G}\right)^{-3 / 2} \exp \left\{\frac{A}{4 G}\right\} .
$$

If we can now choose $\beta$ to be such that $c$ is a universal constant, independent of $A$, we find an entropy

$$
S \sim \frac{A}{4 G}-\frac{3}{2} \ln \left(\frac{A}{4 G}\right)+\text { const. }+\ldots,
$$

in agreement with the result (1.1) of Kaul and Majumdar. 


\section{String Theory}

Much of the current interest in black hole entropy was sparked by the discovery by Strominger and Vafa [15] that for extremal (BPS) black holes in string theory, one could compute the Bekenstein-Hawking entropy by counting D-brane states. The relevant configurations are obtained by compactifying a suitable string theory on a manifold with the topology $M \times S^{1}-M$ is $K 3$ in the case considered in Ref. [15], but may be different for other black holes - and considering a collection of D-branes wrapped around cycles of $M \times S^{1}$. To count states, one takes the radius of the $S^{1}$ factor to be large compared to $M$, and describes the low-energy excitations of the D-branes in terms of fields moving on $S^{1}$. This description involves a weak-coupling approximation, and is unrealistic for a true black hole. For BPS configurations, however, one can argue that the density of states is protected by nonrenormalization theorems when the coupling is increased.

The sigma model describing the excitations on $S^{1}$ is a two-dimensional conformal field theory, albeit a conformal field theory very different from those considered in the preceding sections of this paper. Thus Cardy's formula may be used to count states, and we may again appeal to Eqn. (2.9) to find the logarithmic corrections to the entropy.

For the five-dimensional black holes investigated by Strominger and Vafa, the relevant conformal field theory has central charge

$$
c \approx 3 Q_{F}^{2}
$$

and $L_{0}$ eigenvalue

$$
\Delta=Q_{H},
$$

where $Q_{F}$ is the Ramond-Ramond charge and $Q_{H}$ is the momentum around the $S^{1}$. These quantities translate into charges of the associated black holes, and the entropy obtained from the exponential term in the Cardy formula turns out to be $A / 4 G$, where the horizon area is

$$
A=8 \pi \sqrt{\frac{Q_{H} Q_{F}^{2}}{2}} .
$$

By (2.9), the leading correction to the entropy is thus

$$
S \sim \frac{A}{4 G}-\frac{3}{2} \ln \left(\frac{A}{4 G}\right)+2 \ln Q_{F}+\text { const. }+\ldots
$$

We again obtain a logarithmic correction identical to that of Kaul and Majumdar, along with an extra term depending on the Ramond-Ramond charge.

A similar analysis can be performed for the four-dimensional black holes of Horowitz et al. [16,23], which are obtained from a string theory compactified on a six-torus, with charges $Q_{2}, Q_{5}, Q_{6}$, and $n$ carried by two-branes, five-branes, six-branes, and strings wrapped around cycles of the torus. In the extremal limit, one obtains a conformal field theory with

$$
c=6 Q_{2} Q_{5} Q_{6}, \quad \Delta=n,
$$


corresponding to a black hole with horizon area

$$
A=2 \pi \sqrt{Q_{2} Q_{5} Q_{6} n} .
$$

The leading correction to the entropy is thus

$$
S \sim \frac{A}{4 G}-\frac{3}{2} \ln \left(\frac{A}{4 G}\right)+\ln \left(Q_{2} Q_{5} Q_{6}\right)+\text { const. }+\ldots
$$

Again, we obtain an area term of the form (1.1), plus corrections that depend on conserved charges.

Larsen has proposed a related conformal field theoretical picture for a large class of five-dimensional charged, rotating black holes [12, 24]. Such black holes are characterized by a mass $M$, three conserved charges $Q_{i}$, and two angular momenta $J_{R, L}$. The mass and charges can be parametrized as

$$
M=\frac{1}{2} \mu \sum_{i=1}^{3} \cosh 2 \delta_{i}, \quad Q_{i}=\frac{1}{2} \sinh 2 \delta_{i}
$$

Larsen shows that many of the properties of such black holes can be understood in terms of a left- and right-moving conformal field theory, each with central charge $c=6$, with $L_{0}$ eigenvalues

$$
\Delta_{R, L}=\frac{1}{4} \mu^{3}\left(\prod_{i} \cosh \delta_{i} \mp \prod_{i} \sinh \delta_{i}\right)^{2}-J_{R, L}^{2} .
$$

The Bekenstein-Hawking entropy follows from Cardy's formula, and can be written in the form

$$
S_{B-H}=S_{R}+S_{L}, \quad S_{R, L}=2 \pi \sqrt{\frac{c \Delta_{R, L}}{6}} .
$$

Since the central charge $c$ is just a number, it is easy to use Eqn. (2.9) to obtain the logarithmic corrections to (5.10). Using the results of [12], we obtain

$$
S \sim \frac{A_{+}}{4 G}-\frac{3}{2} \ln \left(S_{L} S_{R}\right)+\text { const. }+\ldots=\frac{A_{+}}{4 G}-\frac{3}{2} \ln \left[\left(\frac{A_{+}}{4 G}\right)^{2}-\left(\frac{A_{-}}{4 G}\right)^{2}\right]+\text { const. }+\ldots,
$$

where $A_{ \pm}$are the areas of the outer and inner horizons. This result may be compared to the nearly identical expression (3.9) for the BTZ black hole.

\section{Speculations}

Although the models of black hole entropy considered here involve very different physical pictures of microscopic states, all use two-dimensional conformal field theory as a crucial tool. This makes it possible to compute the leading logarithmic corrections to the 
Bekenstein-Hawking entropy in a simple and systematic manner. The resulting entropy takes the general form

$$
S \sim \frac{A}{4 G}-\frac{3}{2} \ln \left(\frac{A}{4 G}\right)+\ln F(Q)+\text { const. }+\ldots,
$$

where $F(Q)$ is some function of angular momentum and other conserved charges.

The existence of logarithmic corrections of the form $\ln (A / 4 G)$ is thus a general feature of black hole entropies obtained in this manner. The interesting question is whether the factor of $-3 / 2$ in (6.1), which also appears in the results of Kaul and Majumdar, is also universal.

The problem, of course, is that the charges in $F(Q)$ and the horizon area are not, in general, independent, so there is some ambiguity in the division of the right-hand side of (6.1) into separate terms. To explore this issue further, let us return to the Cardy formula (2.9), and note that it can be rewritten as

$$
\ln \rho(\Delta)=S_{0}-\frac{3}{2} \ln S_{0}+\ln c+\text { const. }
$$

where $S_{0}=2 \pi \sqrt{c \Delta / 6}$. Hence if the entropy is obtained from a single conformal field theory, and if the central charge $c$ is "universal" in the sense of being independent of the horizon area, the factor of $-3 / 2$ will always appear.

This sort of "universality" of the central charge seems to be a sensible requirement for any fundamental conformal field theoretical explanation of black hole entropy: one should surely be looking for a single conformal field theory to describe black holes with arbitrary masses and charges. The conformal theories of Refs. [3, [4, 5] do not satisfy this demand, but they are presumably not yet the final word on horizon boundary conditions.

The requirement of a single conformal field theory is less clear. One might reasonably argue that there should be left-moving and a right-moving sectors, as there are for the asymptotic symmetries of the BTZ black hole. Larsen, for instance, has suggested that these sectors could be associated with the inner and outer horizon [12]. The presence of two conformal field theories changes the form of the leading correction in (6.2), which becomes

$$
\ln \rho\left(\Delta_{L}\right)+\ln \rho\left(\Delta_{R}\right)=S_{L}+S_{R}-\frac{3}{2} \ln \left(S_{L} S_{R}\right)+\ln \left(c_{L} c_{R}\right)+\text { const. }
$$

It is evident that if $S_{L}+S_{R}$ gives the standard Bekenstein-Hawking entropy, the logarithmic correction is no longer $-\frac{3}{2} \ln S_{B-H}$, but rather takes a form more like that of Eqn. (5.11).

This difference appears to account for much of the variation among the logarithmic terms found in this paper. The difference may be related to the choice of how to treat angular momentum and other conserved charges when counting states, and thus implicitly to the choice of which states to count. For the $(2+1)$-dimensional black hole, for example, Strominger's approach starts with a black hole with a fixed angular momentum, and gives an expression of the general form (6.3). The earlier approach of Ref. [21], on the other 
hand, does not require a specified angular momentum, and yields an expression of the form (6.2). It will be interesting to see how the results of Kaul and Majumdar [7], which are based on boundary conditions specific to a nonrotating black hole, change when more general rotating boundary conditions are incorporated.

\section{Acknowledgements}

This work was supported in part by Department of Energy grant DE-FG03-91ER40674.

\section{Appendix A. The String Level Density and the Cardy Formula}

The expression (2.9) for the density of states differs from the "level density" of string theory [25], which counts the oscillator states of a string theory. In this appendix I investigate the differences, while simultaneously obtaining a useful check of the methods of section 2 .

Let us begin by using the procedure that led to Eqn. (2.9) to evaluate the "partition function" $p(n)$ of Ramanujan and Hardy [26], the number of partitions of an integer $n$ into a sum of smaller integers. It is easy to see that $p(n)$ is also the number of oscillator states of a bosonic string in one transverse dimension. The generating function for $p(n)$ is

$$
G(\tau)=\sum p(n) e^{2 \pi i n \tau}=\prod_{n=1}^{\infty}\left(1-e^{2 \pi i n \tau}\right)^{-1}=e^{\frac{2 \pi i \tau}{24}} \eta^{-1}(\tau)
$$

where $\eta(\tau)$ is the Dedekind eta function. This is almost a chiral partition function $Z(\tau)$ for a $c=1$ conformal field theory, but not quite: the modular transformation properties of $\eta(\tau)$ differ slightly from the standard conformal field theory form. Indeed,

$$
\eta^{-1}(-1 / \tau)=(-i \tau)^{-1 / 2} \eta^{-1}(\tau)
$$

so unlike Cardy's partition function (2.4), the quantity $e^{-2 \pi i \tau / 24} G(\tau)$ is not exactly modular invariant. It is straightforward to incorporate the extra factor in the transformation (A.2)

into the integral (2.6), however: one must simply replace $\tilde{Z}(-1 / \tau)$ by $(-i \tau)^{1 / 2} \tilde{Z}(-1 / \tau)$. This translates into a term $f\left(\tau_{0}\right)=\left(-i \tau_{0}\right)^{1 / 2}=(-b / a)^{1 / 4}$ in Eqn. (2.8), giving

$$
p(n) \sim \frac{1}{\sqrt{48} n} e^{\pi \sqrt{\frac{2 n}{3}}}
$$

in exact agreement with the asymptotic results of Ramanujan and Hardy.

The generalization to a partition with $N$ "colors" is immediate. The relevant generating function is now

$$
G_{N}(\tau)=\sum p_{N}(n) e^{2 \pi i n \tau}=\prod_{n=1}^{\infty}\left(1-e^{2 \pi i n \tau}\right)^{-N}=\left(e^{\frac{2 \pi i \tau}{24}} \eta^{-1}(\tau)\right)^{N}
$$


giving a factor of $\left(-i \tau_{0}\right)^{N / 2}$ in Eqn. (2.8) and an asymptotic behavior

$$
p_{N}(n) \sim \frac{1}{\sqrt{2}}\left(\frac{N}{24}\right)^{\frac{N+1}{4}} n^{-\frac{N+3}{4}} \exp \left\{2 \pi \sqrt{\frac{N n}{6}}\right\} .
$$

This can be recognized as the "level density" for a bosonic string in $N$ transverse dimensions, as first computed by Huang and Weinberg in the context of the Veneziano model [27. This expression and its superstring generalization have been used by Solodukhin to examine logarithmic corrections in the string-black hole correspondence [28].

Let us now try to understand the reason for the differences between the densities of states (2.9) in conformal field theory and the level density (A.5). The key observation is that the partition function for a scalar field in conformal field theory is not actually given by Eqn. (A.1), but is rather [29]

$$
Z(\tau)=\tau_{2}^{-1 / 4} e^{\frac{2 \pi i \tau}{24}} \eta^{-1}(\tau)
$$

where the extra factor of $\tau_{2}^{-1 / 4}$ comes from zeta function regularization of a determinant, that is, from zero-modes of the boson. Under the transformation $\tau \rightarrow-1 / \tau$, we have $\tau_{2} \rightarrow \tau_{2} /|\tau|^{2}$, and it is easily checked that the resulting factor of $|\tau|^{2}$ is just what is needed to compensate for the transformation (A.2) of $\eta(\tau)$, restoring the Cardy formula (2.9).

The role of the zero-modes can be explored further by considering a string compactified on a circle of radius $R$. The Virasoro generators $L_{0}$ and $\bar{L}_{0}$ are then [29]

$$
\begin{aligned}
& L_{0}=\frac{1}{2}\left(\frac{r}{2 R}+s R\right)^{2}+N \\
& \bar{L}_{0}=\frac{1}{2}\left(\frac{r}{2 R}-s R\right)^{2}+\bar{N},
\end{aligned}
$$

where $r$ and $s$ are integer-valued momentum and winding numbers and $N$ and $\bar{N}$ are the usual oscillator number operators. The level density (A.3) counts oscillator states alone, implicitly taking $r=s=0$. To incorporate the winding states, we should instead consider the sum

$$
\rho(\Delta, \bar{\Delta}) \sim \sum_{r, s} p\left(\Delta-\frac{1}{2}\left(\frac{r}{2 R}+s R\right)^{2}\right) \cdot p\left(\bar{\Delta}-\frac{1}{2}\left(\frac{r}{2 R}-s R\right)^{2}\right) .
$$

Approximating the sums by integrals, we can write (A.8) in terms of modified Bessel functions $I_{1}(2 \pi \sqrt{\Delta / 6})$ and $I_{1}(2 \pi \sqrt{\bar{\Delta} / 6})$, and it is straightforward to check that the asymptotic behavior of (A.8) is precisely that of a $c=1$ conformal field theory, as given by Eqn. (2.9). The Cardy formula (2.9) can thus be understood as a total density of states, including both the oscillator states counted by (A.5) and the winding states or zero-modes that can contribute to $\Delta$ and $\bar{\Delta}$. 


\section{Appendix B. Corrections to the Central Charge}

As noted at the end of section 2, the central charge in Eqn. (2.9) generally takes the form

$$
c=c_{\text {class }}+\delta c
$$

where the "classical" central charge $c_{\text {class }}$ is already present in the Poisson brackets of the Virasoro generators. The quantum correction $\delta c$ can be evaluated in some models, and can offer an interesting new test of the statistical mechanical picture. For certain black holes in string theories compactified on Calabi-Yau manifolds, for example, the one-loop contribution to $\delta c$ gives rise to an area-independent term in the Cardy formula [30, 31], which can be interpreted in Wald's Noether charge formalism [32 as the entropy due to a curvature-squared term in the effective action. Since the coefficient of this term in the action is independently calculable in string theory, this agreement provides a delicate test of the statistical mechanical formalism. Similarly, it has been argued that the central charge (3.5) for the BTZ black hole should be shifted to

$$
c=\bar{c}=\frac{3 \ell}{2 G}+\beta+\frac{4 G}{\ell} \gamma
$$

for constants $\beta$ and $\gamma$ that depend on details of the relevant conformal field theory [33]. For an appropriate choice of $\beta$, the resulting correction to the Cardy formula reproduces the shift in entropy found in Ref. 22 from a one-loop path integral computation.

There seems to be no reason to expect the quantum corrections $\delta c$ to have any special universal properties. We should therefore ask whether the logarithmic corrections to the entropy described in this paper are really the leading corrections. Although I know of no completely rigorous argument, for large black holes it seems quite likely that they are:

Recall first that the Bekenstein-Hawking formula for entropy can be obtained from quantum field theory in a classical black hole background [34]. Such a result will receive quantum gravitational corrections, of course, but since the curvature near the horizon decreases as the horizon area $A$ increases, one expects these corrections to be small for large black holes. Equivalently, a term in the four-dimensional effective action with $n$ powers of the curvature will have a coupling constant of dimension $[L]^{2 n-4}$, which can multiply at most a factor of $A^{2-n}$. Quantum corrections to the entropy involve $n>1$ powers of the curvature, and should thus be suppressed by powers of the horizon area. Such an effective action picture may fail for small black holes, but it should be approximately valid in the semiclassical regime, that is, for black holes whose horizons are large compared to the Planck scale.

Now consider an expansion of $\delta c / c_{\text {class }}$ in powers of $A$. The leading correction to the entropy will come from the exponent of Eqn. (2.9), yielding

$$
S \sim \frac{A}{4 \hbar G}\left(1+\frac{\delta c}{c_{\text {class }}}\right)^{1 / 2} .
$$


Positive powers of $A$ in $\delta c / c_{\text {class }}$ would dominate this expression for large $A$, leading to a breakdown of the Bekenstein-Hawking formula in precisely the regime in which it should be most reliable. As argued above, such terms are thus unlikely.

The next terms in the expansion, those of order $A^{0}$, certainly can occur. Their main effect in Eqn. (B.3) will be to shift the coefficient $1 / 4 \hbar G$ in the Bekenstein-Hawking formula. But at least for corrections induced by ordinary matter fields, such shifts can be shown to merely correspond to renormalizations of Newton's constant 35. If this is the case in general - as, once again, one would expect from the semiclassical derivations of the Bekenstein-Hawking formula - then the first observable effects of quantum corrections to the central charge will come at order $A^{-1}$ in $\delta c / c_{\text {class }}$.

Such corrections will, for large $A$, give area-independent terms in the entropy of the sort discussed in Refs. [30, 31]. As noted above, these are certainly important as independent tests of statistical mechanical formulations. But for large $A$, they will normally be dominated by the logarithmic corrections to the Cardy formula discussed in this paper.

\section{References}

[1] For a review, see D. Youm, Phys. Rep. 316 (1999) 1.

[2] See, for example, A. Ashtekar et al., Phys. Rev. Lett. 80 (1998) 904.

[3] S. Carlip, Phys. Rev. Lett. 82 (1999) 2828.

[4] S. Carlip, Class. Quant. Grav. 16 (1999) 3327.

[5] S. Carlip, "Black Hole Entropy from Horizon Conformal Field Theory," in Constrained Dynamics and Quantum Gravity 1999, V. De Alfaro et al., editors, Nucl. Phys. B (Proc. Suppl.) 88 (2000) 10.

[6] S. N. Solodukhin, Phys. Lett. B454 (1999) 213.

[7] R. K. Kaul and P. Majumdar, Phys. Rev. Lett. 84 (2000) 5255.

[8] R. K. Kaul and P. Majumdar, Phys. Lett. B439 (1998) 267.

[9] J. A. Cardy, Nucl. Phys. B270 (1986) 186.

[10] H. W. J. Blöte, J. A. Cardy, and M. P. Nightingale, Phys. Rev. Lett. 56 (1986) 742.

[11] J. Maldacena and A. Strominger, Phys. Rev. D56 (1997) 4975.

[12] F. Larsen, Phys. Rev. D56 (1997) 1005.

[13] A. Strominger, J. High Energy Phys. 9802 (1998) 009.

[14] M. Bañados, C. Teitelboim, and J. Zanelli, Phys. Rev. Lett. 69 (1992) 1849. 
[15] A. Strominger and C. Vafa, Phys. Lett. B379 (1996) 99.

[16] G. T. Horowitz, D. A. Lowe, and J. Maldacena, Phys. Rev. Lett. 77 (1996) 430.

[17] S. Carlip, Class. Quant. Grav. 15 (1998) 3609.

[18] R. Dijkgraaf, J. Maldacena, G. Moore, and E. Verlinde, "A Black Hole Farey Tail," hep-th/0005003.

[19] J. D. Brown and M. Henneaux, Commun. Math. Phys. 104 (1986) 207.

[20] M. Bañados, Phys. Rev. D52 (1995) 5816.

[21] S. Carlip, Phys. Rev. D51 (1995) 632.

[22] S. Carlip and C. Teitelboim, Phys. Rev. D51 (1995) 622.

[23] G. T. Horowitz, "Quantum states of black holes," in Black Holes and Relativistic Stars, R. M. Wald, editor ((University of Chicago Press, Chicago, 1998).

[24] D. Kastor and J. Traschen, Phys. Rev. D57 (1998) 4862.

[25] See, for example, M. B. Green, J. H. Schwarz, and E. Witten, Superstring Theory (Cambridge University Press, Cambridge, 1987), Vol. I, section 2.3.5.

[26] S. Ramanujan and G. H. Hardy, Proc. London Math. Soc. (ser. 2) 17 (1918) 75, reprinted in Collected Papers of Srinivase Ramanujan, G. H. Hardy et al., editors (Chelsea Publishing Company, NY, 1962).

[27] K. Huang and S. Weinberg, Phys. Rev. Lett. 25 (1970) 895.

[28] S. N. Solodukhin, Phys. Rev. D57 (1998) 2410.

[29] P. Di Francesco, P. Mathieu, and D. Sénéchal, Conformal Field Theory (Springer, New York, 1997).

[30] J. Maldacena, A. Strominger, and E. Witten, JHEP 12 (1997) 002.

[31] G. L. Cardosa, B. de Wit, and T. Mohaupt, Class. Quant. Grav. 17 (2000) 1007.

[32] V. Iyer and R. M. Wald, Phys. Rev. D52 (1995) 4430.

[33] K. Behrndt, I. Brunner, and I. Gaida, Phys. Lett. B432 (1998) 310.

[34] S. Hawking, Nature 248 (1974) 30.

[35] See, for example, D. V. Fursaev and S. N. Solodukhin, Phys. Lett. B365 (1996) 51. 